\title{
Epidemiology of Neonatal Sepsis and Associated Factors Implicated: Observational Study at Neonatal Intensive Care Unit of Arsi University Teaching and Referral Hospital, South East Ethiopia
}

\author{
Abebe Sorsa
}

\section{OPEN ACCESS}

Citation: Abebe Sorsa. Epidemiology of Neonatal Sepsis and Associated Factors Implicated: Observational Study at Neonatal Intensive Care Unit of Arsi University Teaching and Referral Hospital, South East Ethiopia. Ethiop J Health Sci. 2019;29(3):333.

doi:http://dx.doi.org/10.4314/ ejhs.v29i3.5 Received: December 14, 2018

Accepted: April 4, 2019

Published: May 1, 2019

Copyright: (C) Abebe Sorsa. This is an open access article distributed under the terms of the Creative Commons Attribution License, which permits unrestricted use, distribution, and reproduction in any medium, provided the original author and source are credited.

Funding: Arsi University

Competing Interests: The author declared that this manuscript was approved by all authors in its form and that no competing interest exists.

Affiliation and Correspondence:

${ }^{1}$ Department of pediatrics and child health Arsi University College of Health Science, Asella, Ethiopia

*Email: nathanabebe08@gmail.com

\section{ABSTRACT}

BACKGROUND: Globally, sepsis remains one of the major causes of morbidity and mortality in neonates, in spite of recent advances in health care units. The major burden of the problem occurs in the developing world while most evidence is derived from developed countries. The objective of this study was to evaluate the epidemiology of neonatal sepsis and associated factors among neonates admitted to Neonatal Intensive Care Unit (NICU).

METHODS: Hospital based prospective cross-sectional study was conducted from April 2016 to May 2017. Neonates with clinical sepsis were included into the study. Data were analyzed using SPSS version 20. Frequencies, proportion and summary statistics were used to describe the study population in relation to relevant variables. Multivariate logistic regressions were used to assess factors associated with neonatal sepsis. p-values of $<0.05$ were considered statistically significant.

RESULTS: A total of 901neonates were admitted to NICU of which 303 neonates were admitted with diagnosis of clinical sepsis making the prevalence of neonatal sepsis to be $34 \%$. Bacteremia were confirmed in 88/303(29.3\%) of clinical sepsis, and gram-positive bacteria constituted 47/88(53.4\%). Of all positive blood cultures, 52/88(59.1\%) were reported from late onset sepsis. Coagulase negative staphylococcus (CoNS) accounted for 22/88(25\%) followed by $E$. coli and $S$. aureus, each contributing 18/88(20.3\%) and 16/88(18.2\%) respectively. Prolonged PROM, low fifth Apgar score, prematurity and low birth weight were strongly associated with increased risk of neonatal sepsis. Neonates born to mothers who received antibiotics during labor and delivery were at significantly lower risk of acquiring neonatal sepsis.

CONCLUSION: The prevalence of neonatal sepsis was high, and most causes of neonatal sepsis were gram positive bacteria and most bacteria isolates were from late onset sepsis. Obstetric factors were strongly associated with development of neonatal sepsis. Intrapartal antibiotic administration significantly reduces neonatal sepsis.

KEYWORDS: Neonatal sepsis, gram positive, gram negative, risk factor 


\section{INTRODUCTION}

Neonatal sepsis is defined as a clinical syndrome in an infant of 28 days of life or younger. It is manifested by systemic signs of infection and isolation of a bacterial pathogen from the bloodstream. Neonatal sepsis further classified into early onset neonatal sepsis (EONS) if clinical feature of sepsis occurs within the first week of life and late onset neonatal sepsis (LONS) if it occurs between 7-28 days of life (1-4). Sepsis is one of the major causes of morbidity and mortality in neonates worldwide, in spite of recent advances in healthcare system $(1,2)$. The rates of blood stream infection (BSI) in neonates are 3-20 times higher in developing countries, and in some countries, approximately half of the patients in neonatal intensive care units (NICUs) acquire infection. The case fatality rates of neonatal sepsis may reach $52 \%$ contributing for almost one million deaths and is responsible for about $30-50 \%$ of the total neonatal deaths in developing countries even though sepsis related mortality is largely preventable with prevention of sepsis itself, timely recognition, rational antimicrobial therapy and aggressive supportive care (4-6). Although high burden of neonatal sepsis related mortality and mortality are being reported from developing countries, most scientific evidences are derived from developed countries. In Ethiopia, neonatal mortality accounts for about $40 \%$ of under-five mortality, and sepsis accounts for about $30-35 \%$ of neonatal deaths only preceded by prematurity and its complications (9). On the other hand, the survivors of neonatal sepsis remain vulnerable to short and long-term neuro- developmental morbidity impacting the overall productivity of the child as adult. Neonatal sepsis is primarily caused by diverse species of bacteria, viral and Candida (7-9). This diverse nature of the organisms implicated in causing neonatal sepsis varies from region to region, and changes over time even in the same place which could be attributed to the changing pattern of antibiotic use and changes in lifestyle $(10,11)$. There are a number of risk factors associated with neonatal sepsis. Common risk factors for neonatal sepsis in sub-Saharan
Africa have been identified as prematurity, PROM, maternal pyrexia, low birth-weight and obstructed labor or birth asphyxia which is usually characterized by low first and fifth Apgar score at birth (12).

The objective of this study was to evaluate the epidemiology of neonatal sepsis and factors associated with neonatal sepsis among neonates admitted to neonatal intensive care unit (NICU).

\section{METHODS AND MATERIALS}

Study design: Hospital based prospective crosssectionalstudy was conducted from May 2016 to April2017.

Study setting (area): The study was conducted at Asella Teaching and Rferral Hospital which is one of the federal referral hospitals located in Arsi Zone,Oromia region, South East Ethiopia. The hospital was renovated and restructured as new hospital in 1992 in which it was designed to delivering service for a population close to 3-4 million in its catchment area as reports from zonal health bureaus indicated.

The service deliveries are organized under different departments in which the majorly include Internal medicine, Surgery, Obstetrics-Gynecology, Pediatric and Child health and diagnostic departments. Pediatric and Child health department is one of the major units of the hospital delivering services under four main sub-units which include: Inpatient, NICU and Outpatient units and follow up clinics. NICU is one of the busiest wards of the hospital with annual average admission rate of 1050 . The NICU is rendering service under critical newborn care unit, septic ward, kangaroo mother care (KMC) and mother side. The NICU is staffed with trained nursing staffs, three general practitioners, two pediatricians and one neonatologist. With regard to the equipment and other facilities, it is equipped with adequate number of different sources of oxygen, continuous positive airway pressure (CPAP), bedside cardiac monitor and bedside ultrasound.

DOI: http://dx.doi.org/10.4314/ejhs.v29i3.2 
Source population: All neonates admitted to Neonatal Intensive Care Unit of Assela Teaching and Referral Hospital during the study period were the source population.

Study population: All neonates who fulfilled inclusion criteria constituted the study population.

Inclusion criteria: The study included neonates with clinical diagnosis of sepsis based on the following two risk factors and/or clinical features of bacterial infections $(1,6)$. Risk factors include: Low birthweight $(<2500$ grams) or prematurity ( $<37$ weeks of gestation age), febrile illness in the mother within 2 weeks prior to delivery, foul smelling discharge and/or meconium stained amniotic liquid. Suspected chorioamnionitis, prolonged rupture of membranes $>18$ hours, prolonged labor (sum of 1st and 2nd stage of labor $>24 \mathrm{hrs}$.) Perinatal asphyxia (Apgar score $<4$ at 1 minute). Clinical festuture of sepsis include: (poor reflexes, lethargy, respiratory distress, bradycardia, apnea, fever, convulsions, abdominal distension, and bleeding (1).

Exclusion criteria: Neonates whose mothers were not available for interview to complement the data despite the neonates fulfilling the inclusion criteria were excluded because of possible risk of data incompleteness and inconsistence. Similarly, critically ill newborns who were not able to undergo the necessary laboratory evaluations were also excluded.

\section{Operational definition (8):}

Early onset sepsis: sepsis diagnosed in the first six days of life

Late onset sepsis: sepsis diagnosed between 7 to 28 days of life

Premature rupture of membrane (PROM): rupture of membrane before onset of life

Prolonged Premature rupture of membrane (PROM): PROM lasting for more than $18 \mathrm{hrs}$

Prolonged labor: Total duration of labor for more than $24 \mathrm{hrs}$

Low birth weight (LBW): birth of 1500-2499gm

Normal birth weight: birth weight of 25003999gm

Leukocytosis: White blood cell (WBC) count $>20,000 / \mathrm{ml}$

Presumptive diagnosis of neonatal meningitis: CSF pleocytosis (WBC> 30cell $/ \mathrm{ml}$ )
Sample size determination and sampling technique: Reports from different studies showed that the prevalence of neonatal sepsis ranges from $20-40 \%(1,10,17,25)$. By taking the maximum value of these findings, which is $40 \%$, allowing for $5 \%$ margin of error (d) with $95 \%$ CI $(\mathrm{z} \alpha / 2=1.96)$ using single population proportion (p) formula, sample size calculated was $n=$ $(\mathrm{Za} / 2) 2 \mathrm{P}(1-\mathrm{P}) / \mathrm{d} 2=(1.96)^{2} \quad 0.4(0.6 /(0.05) 2=369$. As the average annual NICU admission is known, finite population correction formula was applied and $10 \%$ non-response rate was considered to get a final sample size of 303. Non-probably sampling was implemented by including all neonates fulfilling the inclusion criteria till the required sample size got saturated.

Data collection method: Demographic and clinical data were enumerated using standardized format.The format was developed through referring different literatures and a standard text book $(1,2,12)$.

Laboratory data, WBC: About 3-5ml of blood was drawn by a trained neonatal nurse after 24 hours of post-natal age for WBC determination and CRP as tests performed shortly after birth is not adequately sensitive and specific for diagnosing neonatal sepsis and the sample was used for both tests. We used Sysmex-Kx-21N coulter machine to determine WBC per the standard operating procedure (SOP). WBC count of $>20,000$ is defined as leukocytosis and count less than 5,000 defined as leucopenia $(4,5)$.

CRP: C-reactive protein was determined after 24 hours of life using qualitative test. The latex reagent was stored at temperature of 2-8 $0 \mathrm{c}$. The test is based on agglutination principle. The latex reagent was shaken gently and one drop was mixed with drop of serum on slide and mixed with mixer. The slide is rocked forth and back for two minutes for observing microagglutination.

Blood culture: Blood cultures are the gold standard test for the diagnosis of blood stream infection and should be performed in all cases of suspected sepsis prior to administration of antibiotics. Accordingly, blood cultures were taken from neonates with clinical diagnosis of sepsis. Under preferably aseptic techniques blood sample was collected. The laboratory

DOI: http://dx.doi.org/10.4314/ejhs.v29i3.5 
technicians wore sterile gloves during the procedure and prepared a patch of skin approximately $5 \mathrm{~cm}$ in diameter over the proposed site of veni-puncture. This area was cleansed thoroughly with $70 \%$ isopropyl alcohol, followed by povidone-iodine, and followed again by alcohol. The skin was allowed to dry for at least 1 minute before venipuncture. One-mL sample of blood was drawn from a fresh veni-puncture site and added to a bottle containing 5-10 mL of blood Agar culture media. The blood cultures were incubated aerobically at $37^{\circ} \mathrm{C}$ and observed daily for consecutive three days for preliminary results by checking the presence of one of the following findings on culture media: hemolysis, air bubbles (gas production), and coagulation of broth (9). At the same time, subcultures were made during three successive days on enriched and selective media including blood agar, chocolate agar, MacConkey agar and mannitol salt agar plates and examined for growth after 24-48 hours of incubation. Showing no growth on the 7th day, blood cultures were reported as sterile. Isolated bacteria were identified using different standard techniques like Gram stain reaction, biochemical reaction properties (Lactase,Catalase,Indolase), morphological and colony characteristics (9). Antimicrobial sensitivity testing was performed by Kirby Bauer diffusion method using Mueller Hinton agar with incubation of $24 \mathrm{~h}$ at $37^{\circ} \mathrm{C}$ according to Clinical Laboratory Standard Institute standards (CLSI) [10]
Data analysis: Data were entered into EPI-INFO version 3.5.1 and then exported to SPSS version 20 for further analysis. Frequencies, proportion and summary statistics were used to describe the study population in relation to relevant variables. Binary and multivariate logistic regressions were used to assess the factors associated with neonatal sepsis. COR and AOR were used to assess the strength of the association with $95 \%$ confidence interval. p-values of $<0.05$ were considered statistically significant.

Ethical consideration: The research proposal was presented and approved by institutional Review Board of Arsi University, and ethical clearance was obtained. Individual verbal consent was obtained from mothers/care-givers of eligible neonates before interviews/laboratory sampling. Confidentiality and privacy was maintained by conducting interviews in separate spaces as much as possible. Benefits of the laboratory test were clearly communicated to mothers/care-givers. Patients whose blood culture results showed positive growth, susceptibility-based antibiotics were started.

\section{RESULTS}

A total of 901 neonates were admitted to NICU. Of them, 303 werea dmitted with the diagnosis of neonatal sepsis making the prevalence of clinical neonatal sepsis 34\%. Males accounted for 198 $(65.3 \%)$ of neonates with clinical sepsis. And $64(21.1 \%)$ were born preterm, 256(84.6\%) were born at the health facilities (hospitals/health center) and $47(15.4 \%)$ were born at home (Table $1)$.

DOI: http://dx.doi.org/10.4314/ejhs.v29i3.2 
Table 1: Demographic characteristics and clinical feature of neonates with sepsis, at Arsi University Asella Teaching and Referral Hospital, Neonatal ICU, April 2016 to May 2017.

\begin{tabular}{|c|c|c|c|}
\hline Characteristic & & Number & Percent $(\%)$ \\
\hline \multirow[t]{2}{*}{ Sex } & Male & 198 & 65.3 \\
\hline & female & 105 & 34.7 \\
\hline \multirow[t]{2}{*}{ Age } & $<7$ days & 185 & 61.2 \\
\hline & $7-28$ days & 118 & 38.8 \\
\hline \multirow[t]{2}{*}{ Mode of delivery } & SVD* & 220 & 72.5 \\
\hline & $\mathrm{C} / \mathrm{S}^{*}$ & 83 & 27.5 \\
\hline \multirow[t]{3}{*}{ Birth Weight } & $<1500 \mathrm{gm}$ & 18 & 5.9 \\
\hline & $1500-2500 \mathrm{gm}$ & 55 & 18.2 \\
\hline & $>2500 \mathrm{gm}$ & 230 & 75.9 \\
\hline \multirow[t]{2}{*}{$\mathrm{ANC}^{*}$} & $<4$ visit & 193 & 63.8 \\
\hline & $>=4$ visits & 110 & 36.2 \\
\hline \multirow[t]{2}{*}{ Duration of ROM* } & $<18 \mathrm{hrs}$ & 221 & 72.8 \\
\hline & $>=18 \mathrm{hrs}$ & 82 & 27.2 \\
\hline \multirow[t]{2}{*}{ Duration of labor } & $<24 \mathrm{hrs}$ & 224 & 73.8 \\
\hline & $>=24 \mathrm{hrs}$ & 79 & 26.2 \\
\hline \multirow[t]{2}{*}{ Place of delivery } & Health facility & 256 & 84.6 \\
\hline & Home & 47 & 15.4 \\
\hline \multirow[t]{2}{*}{ Respiratory distress } & Yes & 200 & 66 \\
\hline & No & 103 & 34 \\
\hline \multirow[t]{2}{*}{ Temperature } & Hypothermia & 112 & 37.1 \\
\hline & Fever & 143 & 47.3 \\
\hline \multirow[t]{2}{*}{ Neonatal reflex } & Intact & 106 & 35.1 \\
\hline & Depressed & 197 & 62.9 \\
\hline
\end{tabular}

$\mathrm{ANC}^{*}$ - Antenatal care, SVD*-Spontaneous vaginal delivery, C/S*-cesarean section, ROM*-Rupture of membrane.

Table 2: Bivariate and multivariate analysis on selected risk factors associated with blood culture proven neonatal sepsis at ATRH, NICU, May 2016 to April 2017.

\begin{tabular}{|c|c|c|c|c|c|c|c|}
\hline \multicolumn{2}{|l|}{ Parameter } & \multicolumn{2}{|c|}{ Culture proven sepsis } & \multirow{2}{*}{$\begin{array}{l}\text { Univariate } \\
\text { COR }((\% \mathrm{CI})\end{array}$} & \multicolumn{3}{|c|}{ Multivariate analysis } \\
\hline & & yes & No & & Pvalue & AOR $(95 \% \mathrm{CI})$ & $P$ value \\
\hline \multirow[t]{2}{*}{ Sex } & Male & $57(29 \%)$ & $141(71 \%)$ & $1.1(0.93-1.27)$ & 0.27 & $1.405(0.769-2.568)$ & 0.234 \\
\hline & Female & $31(30 \%)$ & $74(70 \%)$ & & & & \\
\hline \multirow{2}{*}{$\begin{array}{l}\text { Mode } \\
\text { delivery }\end{array}$} & $\mathrm{C} / \mathrm{S}^{*}$ & $24(22.7 \%)$ & $64(77.3 \%)$ & & & & \\
\hline & SVD* & $64(24.6 \%)$ & $166(75.4 \%)$ & $1.41(0.83-2.38)$ & 0.67 & $1.11(0.565-2.199)$ & 0.755 \\
\hline \multirow{2}{*}{$\begin{array}{l}\text { Gestational } \\
\text { age in weeks }\end{array}$} & $28-36$ & $20(28.6 \%)$ & $49(71.4 \%)$ & $4.05(1.23-9.15)$ & 0.022 & $3.52(1.305-12.048)$ & 0.044 \\
\hline & $37-42$ & $68(23 \%)$ & $180(77 \%)$ & & & & \\
\hline \multirow{2}{*}{$\begin{array}{l}\text { Place of } \\
\text { delivery }\end{array}$} & Home & $12(23.8 \%)$ & $35(76.2 \%)$ & $1.1(0.48-2.39)$ & 0.86 & $0.98(0.344-2.811)$ & 0.976 \\
\hline & $\begin{array}{l}\text { Health } \\
\text { facility }\end{array}$ & $66(25.8 \%)$ & $190(75.9)$ & & & 7yfgfs & \\
\hline \multirow[t]{2}{*}{ Birth weight } & $1-2.49 \mathrm{~kg}$ & $23(31.5 \%)$ & $50(68.5 \%)$ & $2.10(1.07-4.08)$ & 0.007 & $1.3(0.5-3.3)$ & 0.015 \\
\hline & $2.5-4 \mathrm{~kg}$ & $55(24 \%)$ & $175(76 \%)$ & & & & \\
\hline \multirow{4}{*}{$\begin{array}{l}\text { Prolonged } \\
\text { PROM }^{*} \\
\text { Meconium } \\
\text { stained }\end{array}$} & Yes & $33(35.8 \%)$ & $59(64.2 \%)$ & $1.12(1.01-1.4)$ & 0.048 & $2.31(0.94-5.65)$ & 0.007 \\
\hline & No & $55(26.1 \%)$ & $156(73.9 \%)$ & & & & \\
\hline & Yes & $19(30.2 \%)$ & $44(69.8 \%)$ & $1.10(0.90-1.34)$ & 0.067 & $1.9(0.74-4.77)$ & 0.013 \\
\hline & No & $69(22.8 \%)$ & $171(77.2 \%)$ & & & & \\
\hline \multirow{4}{*}{$\begin{array}{l}\text { liquor } \\
\text { Fifth minute } \\
\text { Apgar score } \\
\text { IAP* }\end{array}$} & $0-6$ & $30(42.9 \%)$ & $40(57.1 \%)$ & $1.60(1.13-2.34)$ & 0.009 & $2.10(1.18-3.73)$ & 0.012 \\
\hline & $7-10$ & $49(26.3 \%)$ & $137(73.7 \%)$ & & & & \\
\hline & Yes & $5(10 \%)$ & $45(90 \%)$ & & & & \\
\hline & No & $83(32.8 \%)$ & $170(67.2 \%)$ & $1.23(1.07-1.4)$ & 0.013 & $3.30(1.122-9.708)$ & 0.03 \\
\hline
\end{tabular}

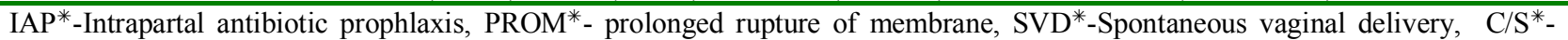
cesarean section

DOI: http://dx.doi.org/10.4314/ejhs.v29i3.5 
Neonates born to mothers with prolonged PROM, preterm delivery had higher risk of neonatal sepsis (Table 2). Similarly, meconium stained amniotic fluid and low birth weight neonates were significantly associated with risk of neonatal sepsis (Table 2).

From the total cases of clinical neonatal sepsis, 185(61.2\%) were recognized as EONS and $118(38.8 \%)$ were recognized as LONS based on the neonates' ages at the time of presentation. About 88/303(29.3\%) of clinical neonatal sepsis were confirmed by blood culture. When the results were disaggregated to the age at presentation, $37(20.0 \%)$ of culture proven cases were from early-onset and 51(43.2\%) were from late-onset sepsis. The rate of culture confirmed neonatal sepsis was significantly higher in LONS than in EONS (AOR 2.8, 95\% CI (1.529-5.080, $P$ $0.001)$. Neonates born from mothers who took antibiotics during labor and delivery were $70 \%$ less likely to develop sepsis than neonates whose mothers didn't take antibiotics (AOR, 0.303; 95\% CI :0.103-0.891, p-0.03).

Laboratory findings: C - reactive protein Results- Positive CRP was documented in 136 neonates with clinical sepsis. Positive CRP strongly predicts culture confirmed neonatal sepsis [AOR 5.71(95\%CI 3.26-10.00), $\mathrm{p}<0.001]$.

White Blood Cell (WBC): WBC was determined for all cases. Abnormal WBC count were found in $99(32.7 \%)$ neonates with clinical sepsis. About $9(2.9 \%)$ cases were having leucopenia (WBC $<5,000 / \mathrm{mm} 3$ ) and $90(29.8 \%)$ were having leukocytosis (WBC $>20,000 / \mathrm{mm} 3)$. The presence of leuckocytosis was 5.7 times more likely in neonates with culture proven sepsis compared to negative results [AOR5.68(95\% CI 3.4159.013) $\mathrm{p}<0.001]$.

Profile of bacterial isolates: Of isolated bacteria, 47(53.4\%) were gram positive bacteria, and most of these isolates were from late onset sepsis (Figure 1). Most blood culture growths were seen among neonates born at heath facilities (hospital and health center). Coagulase negative staphylococci (CoNS) were the most frequently isolated pathogens accounting for $22(25 \%)$, and when further disaggregated to the time of presentation, $17(77.3 \%)$ of isolated CoNS were from neonates with diagnosis of LONS. E.Coli and S.aureus ranked second and third as causes of neonatal sepsis each contributing for about $18(20.3 \%)$ and $16(18.2 \%)$ respectively. Other isolated bacteria were Klebsiella spp, Enterobacter spp and Citrobacter each accounting for $11(12.5), 7(9.7 \%), 5(6.0 \%)$ respectively. No single isolation of group B streptococcus (GBS) was reported in our study. When further disaggregated to specific bacteria gram stain reaction, $80 \%$ of isolated gram positive bacteria were contributed by CONS and S.Aureus while three-forth of isolated Gram negative bacteria were contributed by E.coli and Klebsiella. 


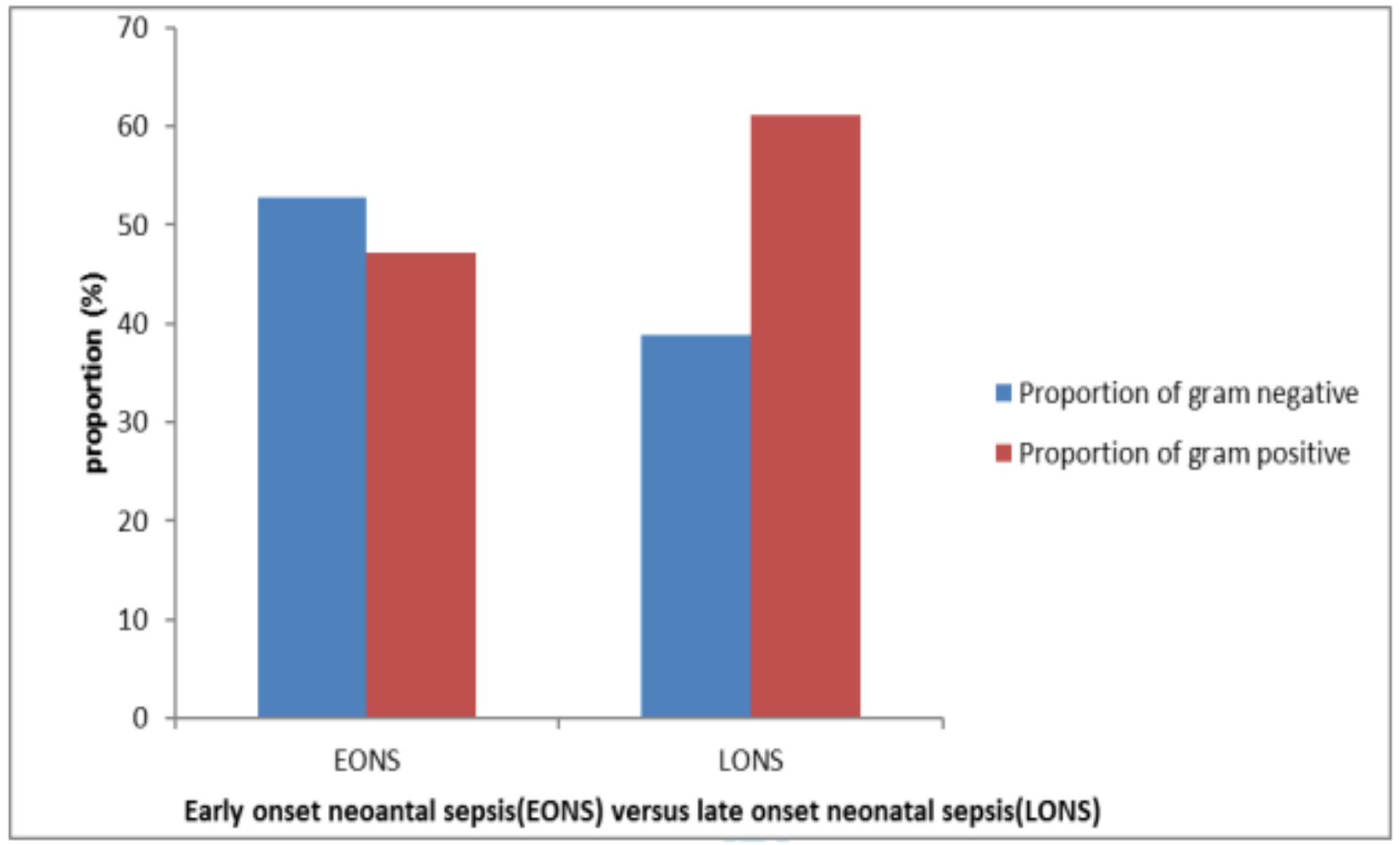

Figure 1: Distribution and patterns of bacterial isolates based on their gram reaction in relation to the age of neonate at presentation at ATRH, NICU, and May 2016 to April 2017.

\section{DISCUSSION}

The prevalence of neonatal sepsis among neonates admitted during the study period was comparable with the national surveillance report. It was also in agreement with the finding from Tanzania which showed prevalence of clinical neonatal sepsis of about 39\%. However, our report was lower than the reports from Egypt which demonstrated prevalence of $45.9 \%$. The difference in prevalence of neonatal sepsis could be explained by the difference in the clinical definition of sepsis at different settings. Some countries like Egypt use more strict criteria to define neonatal sepsis clinically while others use more liberal criteria to define $\operatorname{sepsis}(1,2,3)$. In this study, preterm and low birthweight were strongly associated with risk of developing neonatal sepsis which could be explained by the fact that these groups of newborns had poorly developed immune status (16). On the other hand, neonates born to mothers with prolonged
PROM and Meconium stained liquor were at higher risk of sepsis because of possible ascending colonization of birth canal microorganism and possible aspiration of contaminated meconium stained amniotic fluid. These findings are in line with other studies from different countries which showed low birthweight, preterm, meconium, stained liquor, and prolonged PROM as strong risk factors for neonatal sepsis $(1-7,13,15,16)$.

In this study, babies with low fifth minutes Apgar score was at two-fold risk of acquiring neonatal sepsis. The causes were mainly grampositive bacteria implying that these neonates might need extensive manipulation and resuscitation where they can easily be colonized by these bacteria (15).

The rate of positive blood culture among clinical neonatal sepsis was found to be $29 \%(88 / 303)$ which is comparable with a research finding from Tanzania-Muhimbili which showed growth rate of $24 \%$ but lower than a study from 
Egypt and Tanzania-Mwanza which showed blood culture positivity rate of $40.7 \%$ and $38.5 \%$ respectively $(3,4,24)$. However, the blood culture positivity rate in our study finding was higher than the study from Ghana, Bangalore and other centers which showed blood culture growth rate of $19.2-25.9 \%(1,2,4,5,6,14,27)$. This variation in blood culture positivity rate could be justifiable by the difference in inclusion criteria of the study participants, and technique employed for blood culture processing.

In this study, positive CRP was strongly associated with culture confirmed neonatal sepsis which was in congruent with report from Nigeria and Tanzania in which CRP was an important biochemical test to screen neonatal sepsis. Similarly, this study also demonstrated WBC count of $>20,000 / \mathrm{ml}$ which was significantly associated with culture proven neonatal sepsis. Similar findings were reported from Tanzania and other settings where increased WBC was significantly associated with neonatal sepsis $(10,12,16,18)$

Blood culture growth yield was significantly higher among neonates who presented with LONS than that of EONS which is in agreement with reports from Egypt, Tanzania and other African and Asian countries which showed higher proportion of culture proven sepsis from LONS $(1,2,4,7,9,10,19)$. The justification could be that culture result of neonates with EONS could be affected by maternal antibiotics administration during labor and delivery. Antibiotics administration is the usual scenario at maternity ward. CONS, E. coli, S.Aureus and Klebsiella sppwerethe most commonly identified bacteria isolates in our study which is in line with other study findings from developing countries (1-6, $15,17,20-28)$. Our study did not report any blood culture growth of group $B$ streptococcus (GBS) which is also in congruent with other studies from developing countries where the report was either nil or negligible $(17,19,15)$. This finding could be explained by the fact that GBS is purely acquired through vertical transmission from the mother during labor and delivery where most mother swith risk of GBS colonization receive penicillin antibiotics for which this species of bacteria remain invariably sensitive. Maternal use of antibiotic during labor and delivery was significantly associated with lower risk of neonatal sepsis. Reports from other studies were consistent with our findings $(5,7,12,15)$.

In conclusion, the prevalence of neonatal sepsis was high in this report. The most identified bacteria causing neonatal sepsis were gram positive bacteria and most of which were identified from late onset sepsis. Obstetric risk factors were strongly associated with neonatal sepsis. Intra-partal antibiotic administration significantly reduces neonatal sepsis.

\section{ACKNOWLEDGEMENTS}

I would like to thank Arsi University, College of Health Science, for funding the project and allowing to conduct the study. I would like to extend my heartfelt gratitude to $\mathrm{Mr}$. DegagaWakie (Microbiologists) and other laboratory team members for their immense expert contribution during the study period. Lastly, it is my pleasure to thank Dr.Abayneh Girma for his unreserved constructive inputs to finalize this manuscript.

DOI: http://dx.doi.org/10.4314/ejhs.v29i3.2 


\section{REFERENCES}

1. EmanM.El-Din, Mohamed M. Adel El. Epidemiology of Neonatal Sepsis and Implicated Pathogens. BioMed Research International.2015; 10(6).

2. Neema K, Erasmus K, Damas L M. Predictors of positive blood culture and deaths among neonates with suspected neonatal sepsis in a tertiary hospital, MwanzaTanzania. BMC Pediatrics. 2010; 10(39).

3. Basavaraj M, MrRashmiranjan R and Gagan P. Neonatal Septicemia: Bacterial Isolates \& Their Antibiotics SusceptibilityPatterns: A Hospital Based Study. Journal medical science and clinical research. 2017; 5(6).

4. Appiah-Korang L, Noah Obeng-N, Stephanie B. Neonatal bloodstream infections in a Ghanaian Tertiary Hospital: Are the current antibiotic recommendations adequate? BMC. 2016; 16(598).

5. Shrestha S, Shrestha NC, Dongol Singh S. Bacterial Isolates and its Antibiotic Susceptibility Pattern in NICU. KATHMANDU UNIVERSITY MEDICAL JOURNAL. 2013; 41(1):66-70.

6. Srinivasa $\mathrm{S}$ and Arunkumar D. Bacterial isolates and their Antibiotic susceptibility patterns in Neonatal sepsis.CurrPediatr Res. 2014; 18 (2).

7. DayniaE.B,TrushaN,Charlotte S. Bacterial Bloodstream Infections in Neonates in Developing Country. International Scholarly Research Network pediatrics. 2012; 10(6).

8. Fanaroff and Martin. Postnatal Bacterial Infections. In: Morven S. Edwards. NeonatalPerinatal Medicine. $8^{\text {th }}$ edition. 1600 John F. Kennedy Blvd: MOSBY ELSEVIER; 2008.[p 1123]

9. Shahla A, Mohammad T, Amin S. Trends in Incidence of Neonatal Sepsis and Antibiotic Susceptibility of Causative Agents. Journal of Clinical Neonatology.2012; 1 (3).

10. Barrow GI, Feltham RKA. Cowan and Steel's Manual for Identification of Medical Bacteria. 3rd edition. Cambridge: Cambridge University Press; 2003:219-40

11. Clinical and Laboratory Standard Institute: Performance standards for antimicrobial disk susceptibility tests. Approved standard. In 11th edition document M02-A11. Wayne: Clinical and Laboratory Standards Institute; 2012:12-27

12. Bich-Tram H, Michael P, Benoit G. Burden of bacterial resistance among neonatal infections in low income countries: how convincing is the epidemiological evidence? BMC. 2015; 15(127).

13. Anna C Seale, Michael Mwaniki, Charles R J $\mathrm{C}$ Newton and James A Berkley. Maternal and early onset neonatal bacterial sepsis: burden and strategies for prevention in sub-Saharan Africa.Lancet Infect Dis. 2009; 9(7): 428-438.

14. Flora C, Mariam M Mi, Martha F M. Utility of qualitative $\mathrm{C}$ - reactive protein assay and white blood cells counts in the diagnosis of neonatal septicaemia at Bugando Medical Centre, Tanzania. BMC.2015; 14(248).

15. Tsehaynesh G/e, Feleke M, Setegn E. Bacterial etiologic agents causing neonatal sepsis and associated risk factors in Gondar, Northwest Ethiopia. $\quad B M C$ Pediatrics.2017;17(13).

16. Richard A. Polin. Management of Neonates with Suspected or Proven Early-Onset Bacterial Sepsis. Journal of American Academic of Pediatrics.2012; 129: 1006-15.

17. Barbara J. Stoll and Andi L. Shane. Infections of the Neonatal Infant. In:Robert $M$. Kliegman, Bonita F. Stanton and et al. Nelson Textbook of Pediatrics. $20^{\text {th }}$ ed. Philadelphia: Elsevier; 2015: 909-14

18. Karen M. Puopolo.Epidemiology of NeonatalEarly-onset Sepsis. J.AAP.2008; 9(12).

19. Ramesh Bhat Y, Leslie Edward S L and Vandana KE. Bacterial isolates of early-onset neonatal sepsis and their antibiotic susceptibility pattern between 1998 and 2004: an audit from a center in India. Italian Journal of Pediatrics.2011; 37(32).

20. David Osrin, Stefania Vergnano and Anthony Costello. Serious bacterial infections in newborn infants in developing countries. Lippincott Williams \& Wilkins.2004; 17:21724.

21. Ramesh Bhat $Y$ and Lincy $P$ Baby. EarlyOnset of Neonatal Sepsis: Analysis

DOI: http://dx.doi.org/10.4314/ejhs.v29i3.5 
ofthe Risk Factors and the Bacterial Isolates by Using the BacT Alert System. Journal of Clinical and Diagnostic Research.2011; 5(7): 1385-88.

22. Nyambura M, Heike C and Stephen E. M. Antimicrobial resistance pattern: a report of microbiological cultures at a tertiary hospital in Tanzania. BMC.2016; 16(576).

23. David O, Stefania V and Anthony C. Serious bacterial infections in newborn infants in developing countries. Current Opinion in Infectious Diseases. 2004; 17:217-24.

24. Birju A S. and James F P. Neonatal sepsis: An old problem with new insights. Virulence.2014; 5(1) 170-8.
25. Francesca C, Pietro S, Michele G. Early and Late Infections in Newborns: Where Do We Stand? Pediatrics and Neonatology. 2016; 57(12): 265-73.

26. S Vergnano, $M$ Sharland, $P$ Kazembe, $C$ Mwansambo. Neonatal sepsis: an international perspective. Arch Dis Child Fetal Neonatal. 2005; 90(10): 220-6.

27. Christina W. O, MB ChB, Anna C. S. Empiric Treatment of Neonatal Sepsis in Developing Countries. The Pediatric Infectious Disease Journal. 2015;34(6). 